\title{
Update by the European Food Safety Authority of the dietary reference values for vitamins set for adults in the $\mathbf{E U}$
}

\author{
Celine Dumas ${ }^{1}$, Lucia Fabiani ${ }^{1}$, Sofia Ioannidou ${ }^{2}$, Kristina Pentieva ${ }^{3}$, Dominique Turck $^{4}$ and \\ Monika Neuhäuser-Berthold ${ }^{5}$ \\ ${ }^{1}$ European Food Safety Authority, Nutrition Unit, Scientific Evaluation of Regulated Products Department, \\ Parma, Italy, \\ ${ }^{2}$ European Food Safety Authority, Evidence Management Unit, Risk Assessment \& Scientific Assistance Department, \\ Parma, Italy, \\ ${ }^{3}$ Ulster University, School of Biomedical Sciences, Coleraine, United Kingdom, \\ ${ }^{4}$ University of Lille, Division of Gastroenterology, Hepatology \& Nutrition, Department of Paediatrics, Lille, France \\ and \\ ${ }^{5}$ Justus Liebig University, Institute of Nutrition Science, Giessen, Germany
}

\section{Abstract}

The European Commission requested the European Food Safety Authority (EFSA) to update the dietary reference values (DRVs) for the European Union (EU) previously set by the Scientific Committee on Food (1993). DRVs are science-based reference values for nutrient intake of healthy people. The work on energy, water, macronutrients and vitamins has been completed (2017); that on minerals has continued (2019). We present the derivation of DRVs for 14 vitamins for adults.

To set DRVs for adults, EFSA either used biomarkers of status, a factorial approach, data on health outcomes, or updated EU intake estimates. The latter were initially taken from publications. EFSA then compiled its own food composition database and calculated intake estimates combining it with individual data from representative national surveys from the EFSA Comprehensive European Food Consumption Database.

EFSA set adequate intakes (AIs) for 7 vitamins, and population reference intakes (PRIs) for 7 others, for adults. Based on its review of biomarkers, health outcomes and intake, EFSA confirmed previous DRVs for vitamin K (per kg of body weight), niacin and thiamin (per MJ). Distinct updated values for women and men were derived for vitamins A, B6, C and E. DRVs for vitamins B6 and E were changed to daily amounts (instead of per g of protein or PUFA intake, respectively). Vitamin E was defined as alpha-tocopherol only (instead of alpha-tocopherol equivalents), and the DRV for folate was expressed in $\mu \mathrm{g}$ of dietary folate equivalents (instead of $\mu \mathrm{g})$. DRV for cobalamin became an AI with an increased value compared to the previous PRI. Using updated intake estimates for biotin and pantothenic acid, and intake-status relationship for vitamin D, EFSA set AIs instead of previous acceptable ranges of intake. EFSA increased the PRI for folate, and that for riboflavin for women, considering data on biomarkers, and also those for vitamins $\mathrm{A}$ and $\mathrm{C}$ following a factorial approach. For the first time, a DRV for choline for the EU population was set, based on observed intakes and data on correction of deficiency symptoms.

This review of available evidence led to a substantial update of the previous DRVs for vitamins. It also showed the need for more studies specifically designed to assess nutritional requirements, or to measure food composition (e.g. vitamin K, choline). There is also a need for the development and improvement of biomarkers of intake or status and relevant analytical methods for their use in setting DRVs.

\section{Conflict of Interest}

There is no conflict of interest 